\title{
Servicio educativo: una revisión legislativa de su estructuración frente a las obligaciones del estado
}

\author{
Educational service: a legislative revision of its structuring in the face of the \\ obligations of the state \\ O mentefato conceitual como estratégia de leitura no oitavo grau \\ Andrea Johana Aguilar-Barreto ${ }^{a^{*}}$ \\ a*Doctora en Educación, andreitajaguilar@hotmail.com, ORCID 0000-0003-1074-1673, Universidad Simón
}

Forma de citar: Aguilar, A. J. (2018). Servicio educativo: una revisión legislativa de su estructuración frente a las obligaciones del estado. Perspectivas, 3(2), 110-120.

Recibido: Enero 25 de 2018

Aceptado: Junio 15 de 2018

\section{Palabras clave}

Educación

Servicio educativo

Sistema educativo

Vanguardia educativa
Resumen: En Colombia la Ley 115 de 1994 desde su artículo $3^{\circ}$ menciona que el servicio educativo es prestado por instituciones de carácter público, aunque se permite a los particulares y otras organizaciones del sector solidario fundar establecimientos educativos siempre que se cumplan las condiciones y los requisitos establecidos en la Ley. El presente documento realiza un revisión histórico legislativa de la forma como se ha estructurado de la educación en Colombia, sus distintas modalidades y La estructura curricular que desde el PEI cada institución debe garantizar; de esta revisión documental se devela el compromiso del estado colombiano frente al acceso, la permanencia y la calidad del sistema en su rol de garante de este derecho y servicio público. La presente investigación realiza un análisis del servicio educativo a partir de las realidades del sistema, para finalmente estimar su pertinencia frente a las realidades generacionales.

*Autor para correspondencia

Andrea Johana Aguilar Barreto

andreitajaguilar@hotmail.com 


\section{Keywords}

Education

Educational service

Educational system

educational vanguard

\section{Palavras chave}

Educação

Serviço educativo

Sistema educativo

vanguarda educacional

\section{Introducción}

Ley 115 de 1994 en el parágrafo $3^{\circ}$ del artículo $3^{\circ}$ de la ya citado señala el reconocimiento a la educación como derecho superior, que se impone sobre los derechos económicos que pueden tener las instituciones educativas. Esto demuestra una vez más el espíritu del legislador de la época que tiende a brindarle a la educación un sentido social conforme a su carácter de derecho humano fundamental. Por otro lado la norma permite inferir que la educación no puede ser pensada desde la lógica del mercado y las reglas económicas. Ello no implica que las instituciones educativas privadas no tengan dentro de sus fines la obtención de utilidades, pero dicha meta debe estar en coherencia con la visión social de la educación.

\section{Metodología}

Para Kuhn (1971) los paradigmas son modelos en los que se enmarcan la ciencia, esto es, patrones aceptados en la producción y comprensión del conocimiento; y para Morin (1984) un paradigma se concibe como el conjunto de relaciones y principios lógicos que gobiernan los discursos epistemológicos. En resumen, un paradigma designa la plataforma sobre la cual se interpretan los fenómenos, y dicha plataforma se encuentra integrada por principios, reglas y normas para la práctica científica, pero también por postulados que conllevan a una determinada comprensión razonable de los objetos que se estudian.

Desde un paradigma interpretativo, y como enfoque cualitativo, esta investigación El diseño 
de la investigación es hermenéutico, pertinente para las revisiones teórica-legislativas en este caso del desarrollo del sistema educativo. Este diseño configura un método dialéctico en el que se sigue un proceso entre investigador/lector y texto para la interpretación del discurso o la realidad. En ese orden de ideas, la intención es captar la dialéctica del acontecimiento para entender, comprender y explicar (Cárcamo, 2005).

\section{Resultados}

\section{La estructura de la educación en Colombia}

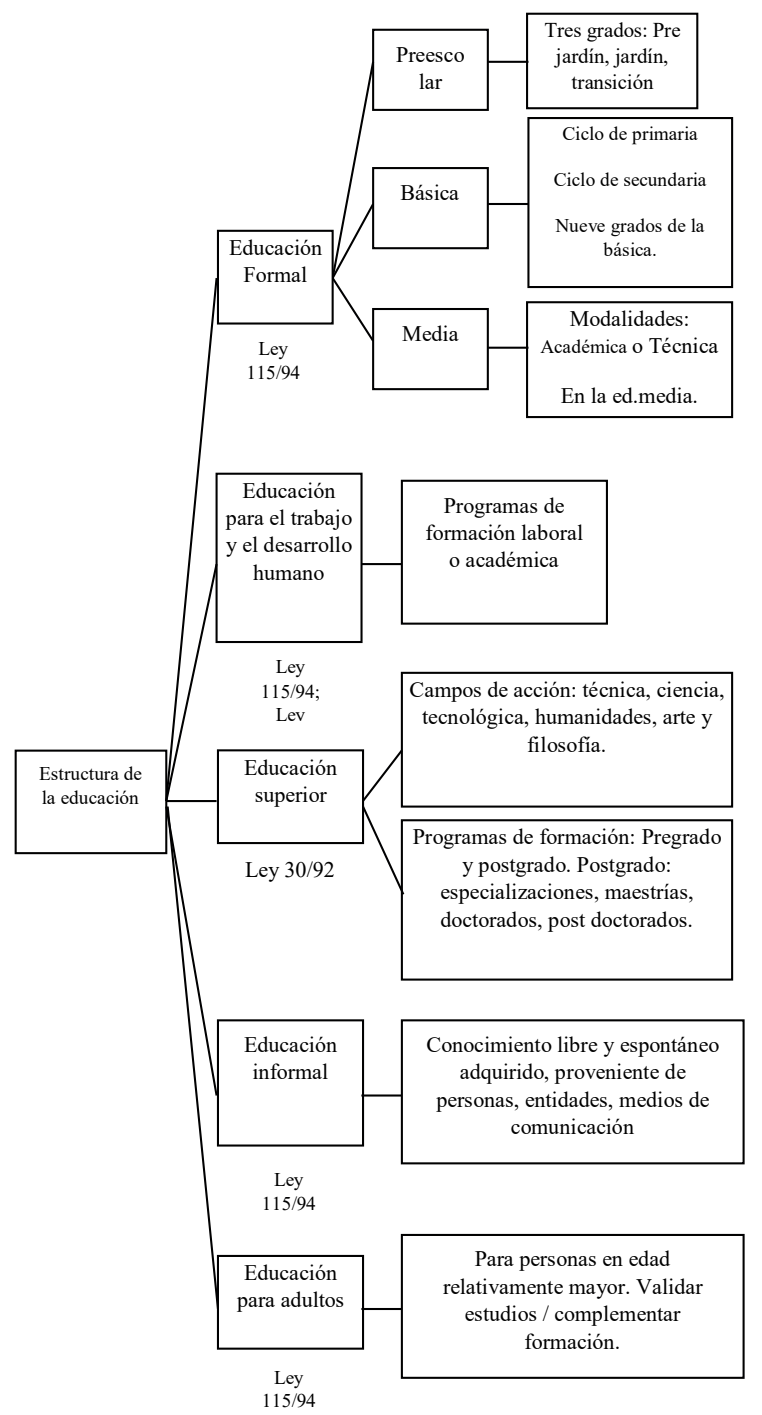

Figura 1. Estructura de la educación en el sistema educativo colombiano Fuente: Aguilar-Barreto (2017)
La educación en Colombia está organizada en cinco grandes bloques: 1. Educación formal, 2. Educación para el trabajo y el desarrollo humano 3. Educación superior, 4. Educación informal, y 5. Educación para adultos. La Figura de La estructura de la educación en Colombia, muestra esta división de la estructura de la educación en Colombia. A su vea la educación formal se estructura en tres niveles: preescolar, básica y media, y a su vez, la primaria se divide en ciclo de primaria y ciclo de secundaria, y la media en la modalidad académica y técnica. Cada nivel está compuesto de grados sumando en total 14. Los objetivos por cada uno de los niveles y ciclos son consignados legalmente, así:

1. Enelartículo 13 delaleygeneralde educación, se establecen los objetivos trasversales para todos los niveles. Los cuales guardan coherencia con los fines de la educación consagrados en el artículo $5^{\circ}$, y se adicionan dos aspectos específicos mediante la Ley 1503 de 2011 (Congreso de la República, 2011, 29 de diciembre) y la Ley 1651 de 2013 (Congreso de la República, 2013, 12 de julio). La primera Ley introduce la educación vial y la segunda incorpora: a) capacidades y habilidades para lograr la igualdad en el acceso a la educación superior y la educación superior; b) habilidades comunicativas básicas en el campo de la enseñanza de la lengua extranjera; y c) requerimiento de certificados de calidad para los programas ofertados por las instituciones de formación para el trabajo y el desarrollo humano. Por otro lado, el artículo 14 de la Ley 115 de 1994 define una enseñanza obligatoria que se gestiona de manera transversal con proyectos lúdicopedagógicos: a) el estudio para la comprensión y la práctica de la Constitución Política, instrucción cívica, paz, solución alternativa de conflictos y derecho laboral, b) el aprovechamiento del tiempo libre, la recreación y el deporte, c) la enseñanza para la protección del medio ambiente y la preservación de los recursos, d) la formación en valores, la justicia, la paz y la democracia, e) la educación sexual, e) hábitos y buenas prácticas en seguridad vial. 
2. En el artículo 15 explica que la educación en el nivel de preescolar La educación preescolar propende por desarrollo integral en las distintas dimensiones biológica, psicomotriz, cognoscitivo, socio-afectivo y espiritual, mediante el desarrollo intencionado de experiencias de socialización pedagógicas y recreativas estructuradas en proyectos. Este tipo de formación se ofrece a la vez se presenta como oportunidad de adaptación. Se dirige a niños menores de 6 años y es obligatorio al menos el grado de transición. Se caracteriza por su aspecto lúdico-pedagógico y recreativo en términos de enseñanza-aprendizaje. Dentro de sus objetivos está el conocimiento del propio cuerpo, el desarrollo de la motricidad y el aprestamiento, el acercamiento a los procesos de lectura y escritura, el desarrollo lógico-matemático, el fomento de la creatividad, el desarrollo de la capacidad para ubicarse en el tiempo y el espacio, la potenciación de la memoria, el fomento de la comunicación y las relaciones sociales, la creación de hábitos alimentarios saludables y de higiene personal, el acercamiento al medio natural y social, entre otros.

3. El artículo 19 señala que el nivel de básica se divide en primaria y secundaria, y busca mediante un currículo común impartir las cuatro áreas fundamentales y de la actividad humana. Por su parte el artículo 20 describe unos objetivos comunes para los dos ciclos, entre los que se encuentran: a) el acercamiento al conocimiento científico, desarrollado desde ámbitos tecnológicos, humanísticos y artísticos, y simultáneamente sus relaciones con la vida social y el entrono b) el desarrollo de habilidades comunicativas básicas -leer, escribir, hablar, escuchar- en lengua materna y lengua extranjera, c) ampliación del razonamiento lógico y analítico para el abordaje de problemas propios de la ciencia, la tecnología y la vida diaria, d) propiciar valores y principios que permitan afrontar los problemas de la realidad nacional -justicia, tolerancia, convivencia, democracia, solidaridad, la cooperación-, e) fomento de la práctica investigativa, f) desarrollo de valores y la moral.
4. El artículo 21 se centra en los objetivos del nivel de asica, específicamente en primaria. Además de los comunes para toda la básica contenidos en el artículo 19, se encuentran de manera específica los siguientes: el fomento por el deseo de aprender y el acercamiento a la realidad social con un sentido crítico, el uso de la lengua como medio de comunicación estética, la asimilación de conceptos científicos de manera coherente con la edad y etapa de desarrollo, la formación para la organización y participación escolar e infantil, la formación artística en los campos de la representación, la expresión corporal, la música, la literatura, las artes plásticas y el desarrollo de habilidades para relacionarse en sociedad. Por otro lado, el artículo 22 se centra en los objetivos del ciclo de secundaria. Además de los expresados en el artículo 19 se incluyen: el desarrollo habilidades comunicativas, entre ellas la lectura comprensiva y critica de diversos textos, entre otros de literatura nacional e internacional; así mismo la resolución de problemas mediante el ejercicio de razonamientos lógicos que derivan del el manejo de los sistemas numéricos, geométricos, métricos, lógicos, analíticos, de conjuntos de operaciones y relaciones con el objetivo de dar solución a los diversos problemas de la cotidianidad, el aprendizaje de los saberes científicos de los fenómenos físicos, químicos y biológicos, el avance en la adquisición de conocimientos sobre la tecnología moderna desde una perspectiva de función social, el estudio científico de la historia nacional e internacional y el estudio del universo y, la tierra, la estructura y organización política y el desarrollo económico.

5. El artículo 27 ofrece al menos tres propósitos generales de la formación media: a) la comprensión de ideas y valores universales, b) la preparación del individuo para su ingreso a la educación superior, y c) la preparación del sujeto para el mundo laboral. De acuerdo a ello la legislación dividió en dos grandes líneas la formación media: la modalidad académica relacionada de forma directa con los dos primeros propósitos y la modalidad técnica relacionada con los dos últimos objetivos. Según los artículos 29 y 30 la modalidad académica tiene como propósito 
profundizar en un campo específico de las ciencias, las artes o las humanidades y acceder a la educación superior, acorde a sus intereses y capacidades, consolidándose desde allí un proyecto de vida y la modalidad técnica tiene como finalidad adiestrar el individuo para que se desempeñe laboralmente en los sectores económicos de producción y de servicios, y para la continuación en la educación superior

6. Los objetivos de la educación media descritos en el artículo 3 para la modalidad académica explicita que se busca: a) profundizar en campos de conocimiento específicos conforme a las capacidades e intereses del estudiante, b) acceder a conocimientos avanzados de las ciencias naturales, c) el desarrollo de prácticas de investigación tanto cualitativa como cuantitativa para el estudio de los fenómenos naturales, económicos, políticos y sociales, d) consolidar la vinculación en programas de desarrollo y organización social/comunitario, e) la participación en acciones cívicas y de servicio social, y f) el desarrollo de la capacidad reflexiva y crítica en los fenómenos.

7. Los objetivos de la educación media en la modalidad técnica se encuentran consagrados en el artículo 33 de la Ley 115 de 1994: a) la capacitación básica inicial para el trabajo; b) la preparación para promover y estimular la vinculación eficiente 1 sector productivo, c) la consolidación de aprendizajes de la educación media académica, que permita al educando el ingreso a la educación superior.

Ahora bien, Lo que en algún momento del desarrollo histórico educativo fue reconocido como la educación no formal, evoluciona, consolidándose como fuente de formación para el trabajo y el desarrollo humano. Esta se ofrece con el objeto de complementar, actualizar, suplir conocimientos y formar, tanto ámbitos académicos como laborales (art. 36, Ley 115 de 1994). Este tipo de educación es regulada por la Ley 1064 de 2006 (Congreso de la República, 2006, 26 de julio).

En este mismo sentido el artículo $2^{\circ}$ puede brindar una perspectiva del origen y la naturaleza de la formación para el trabajo y el desarrollo humano, frente a la cual es Estado le hace un recomiendo como escenarios de formación para el Trabajo y el Desarrollo Humano como factor esencial del proceso educativo de la persona y componente dinamizador en la formación de técnicos laborales y expertos en las artes y oficios. Y es por ello que el Estado patrocina, promueve y respalda las instituciones y programas debidamente acreditados, que forman en este sentido.

Este tipo de educación ha tenido como propósito ampliar las oportunidades de los individuos para acceder al mercado laboral, partiendo de los fundamentos de la teoría del capital humano y la perspectiva que sostiene que el desarrollo sólo es posible en la medida que se genere crecimiento económico. Este tipo de educación ha conducido a que se oferten cientos de programas de formación técnico laboral conforme a las competencias diseñadas por el Observatorio Nacional Ocupacional Colombiano.

Por su parte la educación superior es regulada a través de la Ley 30 de 1992 (Congreso de la República, 1992, 28 de diciembre) y es definida como el proceso de formación permanente que propende por un desarrollo integral desde la promoción del desarrollo de las potencialidades del ser humano; y que como parte de un proceso se ubica necesariamente posterior al ciclo de educación media y tiene por objeto el pleno desarrollo de los alumnos y su formación académica o profesional" (art. $1^{\circ}$ ). Esto permite inferir que la educación superior hace parte de un proceso global y es la cúspide de la formación de los ciudadanos en Colombia. El objetivo de esta formación es el desarrollo pleno del potencial del individuo y se sigue haciendo énfasis en el carácter integral de la misma.

En coherencia, objetivos este nivel educativo tiene la finalidad es moldear a la persona para que pueda brindar una respuesta profesional a los problemas inmediatos de su entorno. Sobre estos aspectos el artículo $4^{\circ}$ describe como independiente a los elementos disciplinares, ésta debe un espíritu reflexivo, orientado al logro de la autonomía personal, en un marco de libertad de pensamiento 
y de pluralismo ideológico que tenga en cuenta la universalidad de los saberes y la particularidad de las formas culturales existentes en el país. Justificándose que su ejercicio se desarrolle en un marco de libertades de enseñanza, de aprendizaje, de investigación y de cátedra.

Otro punto de discusión se puede encontrar en el artículo 2 de la Ley 30: "la Educación Superior es un servicio público cultural, inherente a la finalidad social del Estado". A diferencia de la educación formal en los niveles de preescolar, básica y media, la educación superior tiene un carácter esencial de servicio público y está relacionada con los fines sociales delEstado en coherencia con las obligaciones que se desprenden del Pacto Internacional de los Derechos Económicos, Sociales y Culturales de 1966. De acuerdo a la norma, no todos tienen posibilidad de acceder a la educación superior, pues el artículo $5^{\circ}$ describe que "será accesible a quienes demuestren poseer las capacidades requeridas y cumplan con las condiciones académicas exigidas en cada caso", es decir, se crean muy diversos filtros para no permitir el ingreso al último nivel de la formación en Colombia. Entonces, la esperanza de los individuos de seguir en un proceso de formación de permanente se reduce quedando las vías para otros tipos de formación como la técnica.

Por otro lado, la educación informal conforme al artículo 43 de la Ley General de Educación tiene como particularidad de no estar direccionada por un sistema de niveles o grado. Además, este tipo de educación busca la socialización de un conocimiento particular sin que exija una rigurosidad en la prestación del servicio. Por ello el artículo 43 de la Ley 115 menciona respecto a la educación informal todo conocimiento libre y espontáneamente adquirido, bien sea apropiado de personas, entidades, medios masivos de comunicación, medios impresos, tradiciones, costumbres, comportamientos sociales y otros no estructurados. Como se observa, dicha educación la pueden brindar entidades, personas, medios masivos de comunicación en las diferentes modalidades o provenir de tradiciones, costumbres o comportamientos sociales, por lo que se encuentra muy relacionado con las costumbres de determinadas sociedades.

En este orden de ideas se entiende que en este tipo de educación lo medios de comunicación cumplen una función social, y por ello el artículo 44 de la Ley General de Educación describe que se debe fomentar la participación de los medios de comunicación en procesos de educación permanente y de difusión de la cultura. También describe que el Gobierno Nacional debe diseñar o adoptar mecanismos y estímulos que permitan la adecuada y eficaz utilización de los medios de comunicación masivos como contribución al mejoramiento educativo de la sociedad en general. Por otro lado el artículo 45 crea el Sistema Nacional de Educación Masiva, el cual tiene como propósito de satisfacer la demanda de educación continuada, de validación para la educación formal y de difusión artística y cultural.

Por último, se tiene la educación para adultos, definida por el artículo 50 de la Ley 115 de 1994 como "aquella que se ofrece a las personas en edad relativamente mayor a la aceptada regularmente en la educación por niveles y grados del servicio público educativo, que deseen suplir y completar su formación, o validar sus estudios. Los objetivos de este tipo de educación son cuatro: “a) Adquirir y actualizar su formación básica y facilitar el acceso a los distintos niveles educativos; b) Erradicar el analfabetismo; c) Actualizar los conocimientos, según el nivel de educación, y d) Desarrollar la capacidad de participación en la vida económica, política, social, cultural y comunitaria”. Sin duda alguna la educación para adultos juega un papel fundamental en el desarrollo social del país al permitir que los individuos con edades no permitidas dentro de los niveles de educación formal puedan acceder a conocimientos y saberes básicos. Esto se relaciona con múltiples principios y valores constitucionales, y permite ir cerrando las brechas del analfabetismo y la desigualdad. En efecto el artículo 52 se refiere a la validación en este tipo de educación y menciona que "el Estado ofrecerá a los adultos la posibilidad de validar la educación básica o media y facilitará 
su ingreso a la educación superior, de acuerdo con los requisitos establecidos en la Ley".

Arts. 50-54, Ley 115 de 1994. Se
ofrece a las personas en edad
relativamente mayor a la aceptada
regularmente en la educación por
niveles y grados del servicio público
educativo, que deseen suplir y
completar su formación, o validar sus
estudios.

Figura 2. Modalidades de formación excepcional en el sistema educativo colombiano

Valga aclarar que la educación para adultos se encuentra dentro de las modalidades de atención educativa a poblaciones junto a la educación de personas en situación de discapacidad, la educación para grupos étnicos, la educación campesina y rural, y la educación para la rehabilitación social. Se trata de formas de educación que al igual que la ofertada a los adultos, tiende a la inclusión y a la garantía sin excepción del derecho a la educación para todas las personas.

\section{El proyecto educativo institucional -PEI-}

Indica Alonso (2005) que el PEI ostenta las siguientes características: (a) recrea la identidad de la institución, (b) define fines y objetivos de la escuela, (c) determina las opciones educativas que guían la acción de los docentes e involucrados, (d) es el resultado de la reflexión colectiva de los agentes, (e) permite la puesta en marcha de la institución brindando autonomía y mejora en su funcionamiento, (f) involucra todas las dimensiones y niveles de la escuela, incluyendo el proceso de enseñanza-aprendizaje, la actitud docente, los valores y las actitudes, las capacidades y destrezas, las relaciones entre sus miembros, el sistema estratégico, entre otros.

Lo expuesto permite identificar el PEI como una ruta de planeación o un mapa de navegación para los agentes que lideran y ponen en funcionamiento la acción de la escuela, involucrando a todos los actores o grupos de interés. El horizonte que allí se identifica proporciona sentido y lógica a la acción de la escuela, y con ello se fundamenta la praxis pedagógica, el saber epistémico que circula dentro de este escenario, y la identidad de todos aquellos que hacen parte de la comunidad escolar. Sin duda alguna el PEI esquematiza y pone las bases necesarias para comprender el tipo de educación que se brinda y la calidad que se busca materializar.

En el contexto colombiano, el parágrafo $1^{\circ}$ del artículo 73 de la Ley 115 de 1994 o Ley General de Educación dispone explícitamente sobre el PEI que es un documento de elaboración obligatoria, que entre a apoyar la formación integral. En él se explicitan los principios y los fines que orientan la gestión del establecimiento, los recursos docentes y didácticos disponibles y necesarios, la estrategia 
pedagógica que orienta el que hacer pedagógico, y el reglamento para docentes y estudiantes, dentro múltiples aspectos que direccionan la educación en la institución. (Congreso de la República, 1994, 8 de febrero)

La norma citada pone de manifiesto varios aspectos. Primero el PEI es un medio, un instrumento o un mecanismo del que dispone la escuela y que posibilita la formación del estudiante. Segundo el PEI debe propender por una formación integral, es decir, una formación que implique el desarrollo y el fortalecimiento de las más diversas capacidades y habilidades del ser humano. Sobre este particular, Antúnez, et. Al. (1992, p. 8) menciona que "la educación escolar ha de ser una formación integral y funcional", es decir, una educación que conlleve a la adquisición de capacidades cognitivas, afectivas, psicomotrices, de equilibrio, de autonomía, de interrelación y de inserción social. En consecuencia, los contenidos se convierten en medio y no en fin de la educación, pues se incluyen procedimientos, habilidades, estrategias y valores, normas y actitudes. (p. 8)

Un tercer aspecto que se debe resaltar es que la base del PEI son los principios y fines establecidos pues a partir de estos se organizan los recursos docentes y didácticos, así como la estrategia pedagógica, los reglamentos y los sistemas de gestión. Cuarto el PEI genera autonomía y singularidad a la institución pues esta puede definir su horizonte, perfiles y los medios a requeridos para el logro de los propósitos trazados. Y quinto el PEI es una obligación para cada institución educativa, pues la misma Ley dispone que todas las escuelas deben formular un PEI.

Sin embargo, en la práctica el PEI se ha convertido en tan solo un documento más, un requisito que se asume sin lógica ni funcionalidad. Los agentes educativos revisan los PEI, los modifican, les agregan elementos y lo amplifican, describen muy diversos enfoques pedagógicos, lo transforman para responder a las exigencias de las entidades certificadoras de calidad, pero con todo ello no se genera una transformación de las prácticas pedagógicas ni de la actividad que a diario se evidencia en las aulas. Por tanto, este instrumento es reducido a una formalidad que pierde sentido dentro de la compleja actividad que realiza la escuela a diario.

\section{La estructura curricular en el sistema educativo}

El currículo es asumido desde la legislación educativa colombiana como el conjunto de criterios, planes de estudio, programas, metodologías, y procesos que direccionan hacia la formación integral y a la construcción de la identidad cultural nacional, regional y local, convirtiéndose en medio de materialización del PEI.

\begin{tabular}{|l|l|}
\hline & Dimensión cognitiva/cognoscitiva \\
Dimensión comunicativa \\
Dimensión socio-afectiva \\
Dimensión espiritual \\
& Dimensión ética \\
& Dimensión corporal/psicomotriz \\
& Dimensión estética \\
Ciencias naturales y educación ambiental. & política y democracia. \\
Educación artística y cultural. & Educación ética y en valores humanos. \\
Educación física, recreación y deportes. & \\
Educación religiosa. \\
Humanidades, lengua castellana e idiomas extranjeros. \\
Matemáticas. \\
Tecnología e informática. \\
\hline Ciencias económicas. \\
Ciencias políticas. \\
Filosofia. \\
Ciencias naturales y educación ambiental. \\
Ciencias sociales, historia, geografía, constitución \\
política y democracia. \\
Educación artística y cultural. \\
Educación ética y en valores humanos. \\
Educación física, recreación y deportes. \\
Educación religiosa. \\
Humanidades, lengua castellana e idiomas extranjeros. \\
Matemáticas. \\
Tecnología e informática. \\
\hline
\end{tabular}

Figura 3. Dimensiones o áreas obligatorias de formación por niveles en la educación formal 
En otras palabras, el currículo organiza y estructura la forma en que se materializan los objetivos de la formación para los diferentes niveles y ciclos, y ello exige la definición de planes de estudio, metodologías, criterios de formación y evaluación, medios y recursos, procesos, entre otros. En este aspecto deben considerarse las áreas obligatorias de formación o dimensiones de formación, los cuales pueden ser identificadas en los artículos 15, 23 y 31 de la Ley General de Educación, expuestas en la gráfica anterior.

\section{El acceso, la permanencia y la calidad del sistema educativo colombiano}

Como exponen Aguilar-Barreto, Velanda, Aguilar-Barreto y Rincón (2017) tres elementos esenciales dentro del sistema educativo colombiano son el acceso, la permanencia y la calidad del sistema. Estos componentes son condiciones para la prestación del servicio educativo, pues en palabras del Ministerio de Educación Nacional la educación debe prestarse mediante un servicio público equitativo, es decir, "cumplir con las condiciones que permitan a todos los niños, niñas y jóvenes acceder, permanecer y recibir una educación de calidad en condiciones de igualdad" (Ministerio de Educación Nacional, 2009, p. 15).

El acceso está referido esencialmente al registro y matricula de los niños, las niñas y los jóvenes dentro del sistema educativo, lo cual se relaciona con cobertura y gratuidad. Dentro del sistema educativo colombiano, al menos en los primeros niveles de educación formal, la gratuidad se convierte en el principal factor de la cobertura. Esto tiene relación directa con la obligación del Estado de garantizar la educación a todos los habitantes del territorio, lo cual supone la adopción de estrategias y programas que permitan reducir los obstáculos de ingreso al sistema. Partiendo de la difícil situación económica del país y la mayoría de las familias, además de las marcadas brechas socio-económicas entre grupos sociales, la gratuidad se convierte en un elemento fundamental para el cumplimiento del componente de acceso. Sin embargo, dicha gratuidad no es absoluta, pues "en aras de la solidaridad social, la gratuidad no impide el cobro de los derechos académicos a quienes puedan sufragarlos" (Ministerio de Educación Nacional, 2009, p. 16).

El acceso en el sistema educativo colombiano es un tema amplio de discusión pues la gratuidad no sólo permite el acceso. Además de ello se debe considerar otro tipo de elementos que asociados con el criterio económico y de recursos puede reducir la posibilidad de acceso de un niño o joven al sistema. Así se deberá considerar la perspectiva de desarrollo humano propuesta por Sen (2010) y Sen (2000), la cual entra a considerar las condiciones y posibilidades reales de los sujetos para realizar aquello que valoran. Al reducirse estas capacidades y oportunidades reales, se puede afirmar que se obstaculiza el proceso de desarrollo humano. Y en efecto para nadie es un secreto que las distancias, la falta de recursos, la imposibilidad de las familias de invertir en recursos educativos, entre otros, pueden afectar el acceso a la educación de los niños y jóvenes. También se deberá considerar otros factores como la violencia, el conflicto armado y otros problemas que pueden reducir las capacidades reales de los sujetos.

El segundo componente a considerar dentro del sistema educativo colombiano es la permanencia. Esta se define como la posibilidad de todas las personas de acceder y encontrarse dentro del sistema hasta que culminen sus estudios. Dentro del documento del Ministerio de Educación Nacional (2009) se muestran algunos factores asociados con la deserción escolar en Colombia. La deserción es un fenómeno que tradicionalmente se había asociado a la ruralidad, hoy el concepto evoluciona relacionándose a las condiciones sociales del contexto, a las dificultades de inclusión educativa, a la baja efectividad y pertinencia de las prácticas pedagógicas

Por último, el concepto de calidad ha abierto la puerta a una amplia discusión. Desde el Ministerio de Educación Nacional (2009, p. 18), la calidad educativa "supone que todas las personas pueden 
adquirir y ampliar competencias que les faciliten convivir pacífica y productivamente, indistintamente de su condición social, económica o cultural". Y la discusión se debe a la amplia evidencia que demuestra que el sistema educativo no ofrece una formación equitativa y en igualdad de condiciones para todos los ciudadanos. Ahora bien, la forma como se ha venido garantizando la calidad no ha alcanzado los objetivos trazados.

\section{Consideraciones finales}

En el marco del modelo neoliberal, como lo explican Aguilar-Barreto y otros (2018) se han implementado innumerables estrategias para el fortalecimiento y expansión de discursos y prácticas, cuyo propósito es la socialización y apropiación de representaciones y símbolos que permitan la adaptación de los sujetos al sistema económico imperante. En este escenario, la educación también ha sido impregnada de dicho discurso con el objetivo de reproducirlo a partir de la implementación de programas y proyectos con miras a la formación de sujetos aptos para el trabajo, los procesos productivos y el consumo. Para el logro de estos objetivos se han diseñado y ejecutado diferentes políticas públicas en el campo de la educación, a fin de dar respuesta a las exigencias de los organismos multilaterales. Por lo anterior es factible afirmar que la educación ha servido de engranaje del sistema económico, permitiendo el logro de sus objetivos y metas. Una educación que ha pasado de ser un proceso de socialización a convertirse en una institución de control para la formación de sujetos aptos para el mercado y los intereses de las empresas, es decir, un instrumento del proyecto neoliberal con aquiescencia del Estado a través de políticas educativas.

En las anteriores páginas se pudo observar que el sistema educativo colombiano, esto es la organización de la enseñanza en el país, tiene su origen en la Ley 115 de 1994 y la Ley 30 de 1992, las cuales fomentan en gran medida una formación integral y permanente, así como una la labor educativa que permita el fortalecimiento de múltiples dimensiones en el individuo: lo cognitivo y cognoscitivo, lo emocional, lo comunicativo, lo social y cultural, lo político y ciudadano, la estética y las artes, lo económico y laboral, entre otros. Sin embargo, se puede señalar que el mismo sistema ha sucumbido al direccionamiento del neoliberalismo a partir de políticas educativas que han surgido con posterioridad.

\section{Referencias}

Aguilar-Barreto, AJ. (2017) La Educación en Colombia: Históricamente elemento jurídico político para el desarrollo social. En: Derechos humanos desde una perspectiva sociojurídica, Publicaciones Universidad del Zulia, Maracaibo, estado Zulia, República Bolivariana de Venezuela. Recuperado en: http://bonga. unisimon.edu.co/handle/123456789/2097

Aguilar-Barreto, AJ.; Velandia, Y.; Aguilar-Barreto, CP. y Rincón, G. (2017) Gestión educativa: tendencias de las políticas públicas educativas implementadas en Colombia. Revista Perspectivas Volumen 2 (2), páginas 6-16.

Aguilar-Barreto, AJ.; Velandia, Y.; Aguilar-Barreto, CP. y Rincón, G. (2017) Gestión educativa: tendencias de las políticas públicas educativas implementadas en Colombia. Revista Perspectivas Volumen 2 (2) Julio- Diciembre de 2017, páginas 6-16.

Aguilar-Barreto, AJ; Rodríguez, G. y AguilarBarreto, CP. (2018) Gestión de políticas públicas educativas: Una caracterización en Norte de Santander. Revista Espacios, Vol. 39 ( ${ }^{o}$ 30) Año 2018. Pág. 11. Venezuela. Recuperado en: http://www.revistaespacios. com/a18v39n30/18393005.html

Alonso, J. Manual para elaborar el proyecto educativo de la institución escolar, México, Plaza y Valdés, 2005.

Antúnez, S., Martín, L., Imbernon, F., Parcerisa, A. y Zabala, A. (1992). Del proyecto educativo a la programación en el aula. Barcelona: Graó. 
Callejo, Gómez y Casado (2013). El techo de cristal en el sistema educativo español. Madrid: UNED.

Cárcamo, H. (2005). Hermenéutica y análisis cualitativo. Cinta Moebio, 23, 204-216.

Colombia. Congreso de la República (1992, 28 de diciembre). Ley 30 [Ley de Educación Superior]. Diario Oficial No. 40.700, del 29 de diciembre de 1992.

Colombia. Congreso de la República (1994, 8 de febrero). Ley 115 [Ley General de Educación]. Diario Oficial No. 41.214, del 8 de febrero de 1994.

Colombia. Congreso de la República (2006, 26 de julio). Ley 1064. Diario Oficial No.46.341, del 26 de julio de 2006.

Colombia. Congreso de la República (2011, 29 de diciembre). Ley 1503. Diario Oficial No. 48.298, del 30 de diciembre de 2011.

Colombia. Congreso de la República (2013, 12 de julio). Ley 1651. Diario Oficial No. 48.849, del 12 de julio de 2013.

Kuhn, T. (1971). The estructure of the Scientific Revolutions. Chicago: University Press. Chicago

Ministerio de Educación Nacional (2009). Organización del sistema educativo. Conceptos generales de la educación preescolar, básica y media. Guía No. 33. Bogotá: MEN.

Morin, E. (1984). Ciencia con consciencia. Anthropos. Primera edición. Barcelona. 